\title{
Hybrid Algorithm using the advantage of ACO and Cuckoo Search for Job Scheduling
}

\author{
R.G. Babukartik ${ }^{1}$, P. Dhavachelvan ${ }^{1}$ \\ ${ }^{1}$ Department of Computer Science, Pondicherry University, Pondicherry, \\ India \\ $\{r . g$. babukarthik, dhavachelvan\}@gmail.com
}

\begin{abstract}
:
Job scheduling is a type of combinatorial optimization problem. In this paper, we propose a Hybrid algorithm which combines the merits of ACO and Cuckoo Search. The major problem in the ACO is that, the ant will walk through the path where the chemical substances called pheromone is deposited. This acts as if it lures the artificial ants. Cuckoo search can perform the local search more efficiently and there is only a single parameter apart from the population size. It minimizes the makespan and the scheduling can be used in scientific computing and high power computing.
\end{abstract}

Keywords: Job Scheduling, Ant Colony Optimization, Cuckoo Search.

\section{Introduction}

Job scheduling problem has a combinatorial optimization problem. Job scheduling is used in compound equipment manufacturing system for authenticating the performance of heuristic algorithms. The major problem in job scheduling is that many scheduling do not fit into a common description model. Hence for scheduling problems it is too difficult to define a common frame work.

In this paper, we have proposed a Hybrid algorithm which combines the advantage of ACO and Cuckoo Search so as to solve the job scheduling problems. Job Scheduling can be used in scientific computing and high power computing for solving all the combinatorial optimizations problems. Our approach is based on heuristic principles which have the advantage of minimizing the makespan.

The main contribution of this paper is,

- The proposal of a Hybrid algorithm which combines the advantage of ACO and Cuckoo Search.

- The evaluation of the Job creation time, Task creation time, Result retrieval time and destruction time.

- The performance comparison of the Hybrid algorithm and Ant colony optimization.

\section{Related Work}

The predefined collection of tasks can be performed by JSSP with the allocation of required resources within a given time period of time. Combining the PSO and ACO an approach for computational intelligence technique for solving JSSP is framed. The main objective is to 
minimize the makespan and the waiting time, in such a way the processing order of the job each and every machine is scheduled [1]. Power consumption of the system can be reduced using ACO with the proper allocation of tasks. When the size of the problem increases, a problemspecific optimization is used. By exploring the different solution for mapping and scheduling of the tasks and communication, using ACO it reduces the execution time of the application. Renewable and non-renewable resources are considered, for the heterogeneous embedded systems no formulation of ACO for concurrent mapping and scheduling. It is problem specific optimization,

Optimized performance is not achieved [2]. Using Local Node Fault Recovery (LNFR) the failed subtask execution can be resumed from the interrupted point which is used for fault recovery strategies in local situations. Using ACO multi objective task scheduling can be performed. LNFR can be used in life time of subtasks, number of recoveries to be performed, and grid service reliability models. Proposed a optimization model to minimize the total cost and to maximize the grid service reliability for task scheduling. Since gird has one RMS, it can be viewed as star topology which is very difficult for the complexity of grid [3]. Considered a scheduling model with two agent and batch processing machines with non-identical job. Consideration of the two agents is to minimize the completion time. Product allocation and resources distribution can be performed better with the help of improved ACO algorithm. In batch processing jobs from the same agent have their priority to scheduled, not focused on dynamic arrival of jobs [4].

Mixed-Integer Linear Programming (MILP) model for job shop scheduling solves the problem in mean weighted flow time, the sum of the weighted tardiness and the earliness costs. To find the pareto optimal solution for a given problem, they used a character of scatter search (SS) to select new swarm in each iteration. PSO has the good performance and computational cost and it is also very easy for implementation. The major disadvantage is that they used genetic operators and character of scatter search [5]. Local search can be performed efficiently with the help of PSO and global search is performed using genetic algorithm. Extended compact Particle Swarm Optimization (EcPSO) is developed by combining the benefits of genetic algorithm with PSO. Without losing the unique features they combined the PSO and Estimation distribution algorithm. Algorithm solves the deceptive and symmetric problems. Algorithm does not focus on continuous problems [6]. Wang-Mendel based on PSO is proposed. High-dimension non-linear optimization problems can be solved using PSO. A complete fuzzy rule set has been obtained through extrapolating using modified PSO algorithm to optimize the fuzzy rule centroid of the data covered area. [7]. The major disadvantage of PSO is the premature convergence which can be solved by Intelligent Dynamic Swarm (IDS). It uses the feature selection technologies [8]. Performance of PSO is better in search quality and efficient than the traditional evolutionary heuristics [9].

A Rotary Chaotic Particle Swarm Optimization (RCPSO) algorithm optimizes the scheduling performance in a multi-dimensional complex space. To optimize the grid workflow scheduling in discrete space a novel RD rule is presented to help the PSO. In multi-dimensional complex space the scheduling performance is optimized [10]. SamACO is a way to sample promising variable values in the continuous domain and to use pheromones to guide ant's construction behavior. This has been developed to extend ACO to a continuous optimization [11].ACO algorithm belongs to the constructive method and hence solution for the combinatorial optimization problem is built step by step without backtracking. Further ACO algorithm adds solution components until a complete solution is generated [12]. Due to stochastic optimization nature of the ACS algorithm, only statistical conclusions can be made [13]. 


\section{Problem Definition}

For solving combinatorial optimization problem, an efficient algorithm is necessary. In this section, we have proposed a hybrid algorithm for job scheduling which will combine the advantages of ACO and Cuckoo search.

Problem can be defined as follows. We have $\mathrm{N}$ jobs and $\mathrm{M}$ machines. Each and every job has its own order of execution that has to be performed on $\mathrm{M}$ machines. Each job has its own starting time. The objective of this algorithm is to minimize the makespan and it can also be used for job scheduling in scientific and high power computing.

Some of the assumptions for the job scheduling problem are,

1. Jobs should be finite set.

2. Each and every job contains a series of operations that needs to be performed.

3. Machines should be finite set.

4. All the machines are capable of handling only one operation at a time.

Some of the constraints are,

1. No job should visit the same machines twice.

2. No condition among operation of various jobs.

3. Preemption type of operation is not allowed.

4. A single machine is capable of handling individual job at a time.

5. No machines fail during its operation.

Table 1. PARAMETERS

\begin{tabular}{|l|c|}
\hline \multicolumn{2}{|c|}{ Parameters } \\
\hline \multicolumn{1}{|c|}{ Index } & Totation \\
\hline Input Variables & Processing time \\
\hline Output variables & 32 \\
\hline No. of Tasks & 4 \\
\hline No. of Machines & $T_{c t}$ \\
\hline Task creation time & $t_{s i n f}$ \\
\hline $\begin{array}{l}\text { Time to save disk } \\
\text { information }\end{array}$ & \\
\hline creation time & \\
\hline
\end{tabular}


International Journal of Information Technology Convergence and Services (IJITCS) Vol.2, No.4, August 2012

\section{Proposed System}

\subsection{Hybrid algorithm}

It combines the advantages of Ant Colony Optimization and Cuckoo search. The major disadvantage in the ACO is that while trying to solve the combinatorial optimization problems the search has to performed much faster, but in ACO ant will walk through the path where the chemical substance called pheromone has been deposited. This acts as if it lures the artificial ants. Hence local search will be performing at the faster rate than in the ACO. In order to overcome the above drawback, Cuckoo search is used. Moreover in the Cuckoo search there is only a single parameter apart from the population size.

1) Scheduling Algorithm

Step 1: Initialization - Job creation time, starting time.

Step 2: Find out the number of task $T$ that need to be scheduled.

Step 3: Schedule the task T using a Hybrid algorithm.

Step 4: Assign the task $T$ to the scheduler, using parallel computation to compute the tasks with the help of the workers.

Step 5: Find out the Job creation time $\boldsymbol{T}_{o t}$, Task creation time $\boldsymbol{T}_{\mathrm{or}}$, Result retrieval time $\boldsymbol{R}_{\text {Tt }}$, Job Destruction time $J_{d t}$ and Total time $T_{t}$.

Step 6: Termination check - When the entire task $T_{t}$ has been assigned to the scheduler, the algorithm terminates. Else go to step 2 for scheduling the tasks.

In the former steps, Step 3 is the main process of the algorithm.

Figure 1. Scheduling Algorithm

2) Ant colony Algorithm

Step 1: Initialization

Initialize the pheromone trails, Ant solution construction.

Step 2: Construction

For each ant in each step, choose the number of task that is needed to be scheduled.

Step 3: Local search

Perform the local search using cuckoo search and then update the path.

Step 4: Pheromone update

For each path, compute fitness value and update pheromone.

Step 5: Termination condition

If total_iteration < max_iteration go to step 2 otherwise terminate. 


\section{3) Algorithm for cuckoo search}

Step 1: Initialization

Initialization of nests and random initial solution.

\section{Step 2: Evaluation}

Get the current best nest.

Step 3: Loop construction

While (fmin > Max generation)

Get the cuckoo value by random walk, if not replace it by Levy's flights.

Step 4: Evaluation

Evaluate the quality fitness $F n_{j}$.

Randomly choose nest among $n$, say $j$.

Step 5: Condition

If $\left.F n_{i}>F n_{j}\right)$

Replace $j$ value by new solution.

End

\section{Step 6: Solution construction}

Retain the best solution and nests.

Rank the solution and nests to choose the best.

Pass to next generation.

End while, else go to step 2.

Figure 3. Cuckoo search

\subsection{Flow chart}

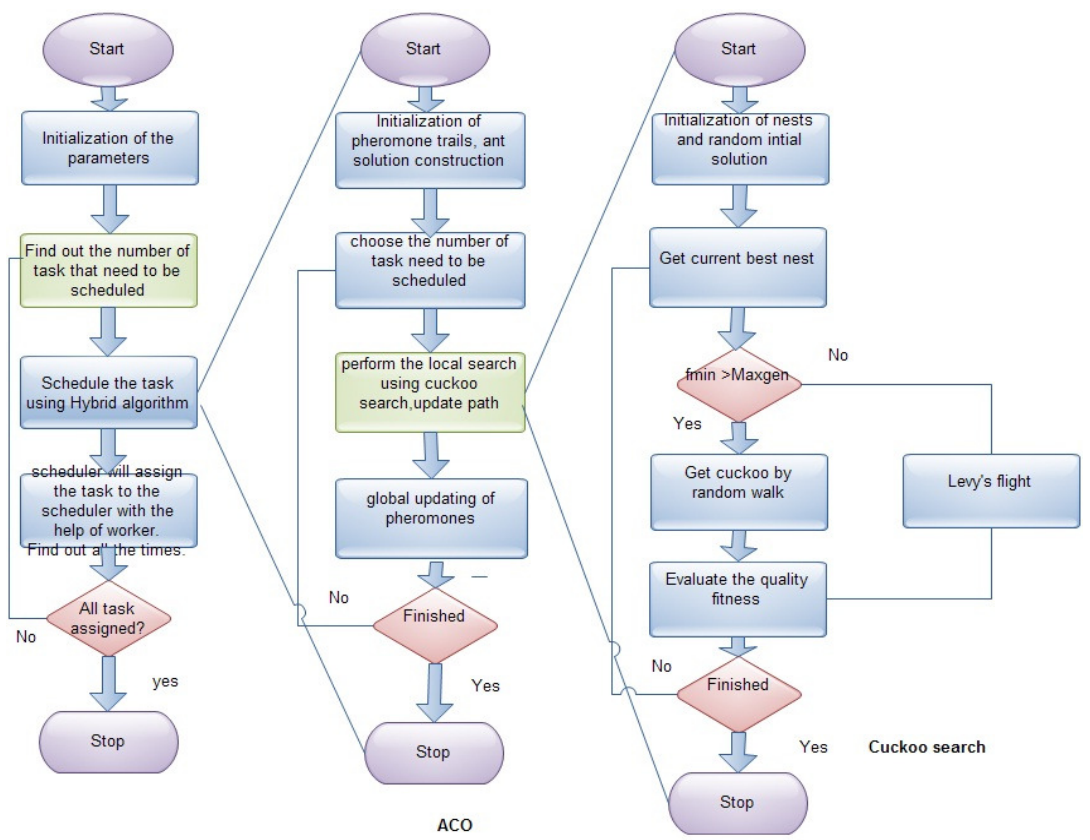

Figure 4. Scheduling flow chart 
Figure 5 shows the flow chart of the Hybrid algorithm, thus the intialization of the parameter that need to be scheduled is performed. Then the number of task that has to be scheduled is identified. Scheduling of resource to the task is carried out with the help of Hybrid algorithm. Once the resource are assigned, the scheduler will assign the task to the worker and the execution of task time is calculated. Hybrid algorithm uses the ACO and Cuckoo search.

ACO Flow chart description: Initialization of the pheromone trails, and ant solution construction is performed. The task that need to be scheduled is choosen, assigning of the resource to the task is performed by Cuckoo search. Global upadation of the resource to the task is performed using the pheromone global update. Termination of task is carried out till the execution of the task is completed.

Cuckoo search Flow chart description: Initilization of the nests and the random initial solution is performed. The current best nest is choosen by the random walk and then the evalution of the qulaity is fitness is performed. Else apply levy's flight for the evluation. Execution is carried out till all the solution is constructed.

\section{Experimental Results}

The performance of the Hybrid algorithm is stimulated using the parallel computing toolbox in matlab with the help of workers. The input value for the scheduling is given in form of number of tasks and the various values are Task creation time and the Result retrieval time. They are obtained in terms of time (seconds). Thus when the number of tasks is increased, the time taken for the creation of tasks is also increased. Result retrieval time for the number of tasks is also increased when the number of tasks is increased. It is obvious from the above result that in other types of algorithm such as PSO and ACO, when the number tasks is increased the time taken for the creation of each and every task is also considerably more, similarly for the result retrieval time will also be more. Where as in hybrid algorithm time taken for the creation of tasks is slightly increased.

\subsection{Task creation Time}

Task creation time can be defined as the time taken to create and save the disk information. Thus job manager saves the task information in its database, for the other types of schedulers, saves the task information in files on the file system.

$$
T_{c:}=c_{z}+t_{\sin f}
$$

Table 2 shows the Task creation time for the various tasks that has been given as the input, for the tasks 1 five task have been created; similarly for the tasks 2 and tasks 5 the corresponding tasks have been created. We took the average for the tasks1, tasks 2 and tasks 4 . Figure 5 show that when the number of tasks increases the task creation time also increases with respect to the time in seconds. Hybrid algorithm shows clearly that task creation time for each and every task increase. 


\subsection{Result Retrieval time}

Result Retrieval time is the time taken to display the result to the client. Generally for the job manager this includes the time taken to obtain the results from the database. For the other types of schedulers is the time taken to read from the file system.

$$
R_{r z}=D_{j n}
$$

Table 3 shows the result retrieval time for the various tasks that has been created, for the tasks 1 five job have been created and 5 result retrieval time has been evaluated based on the time for the task; similarly for the tasks 2 and tasks 4 the corresponding result retrieval time have been found. We took the average for the tasks1, tasks 2 and tasks 4 . Figure 6 show that when the number of tasks increases the result retrieval time also increases with respect to the time in seconds for the job that have been created. Hybrid algorithm shows clearly that result retrieval time for each and every task.

\subsection{Total time}

Total time is the time taken to perform the Job creation time, Task creation time, Job submission time, Job waiting time, Task execution time, Result retrieval time, Job destruction time.

$$
T_{t}=J_{c t}+T_{c t}+J_{s t}+J_{w t}+T_{E t}+R_{r t}+J_{d t}
$$

The table 4 clearly shows the total time taken for the execution of tasks for the job that have been given has the input, thus for 64 task that have been created the corresponding execution time have been evaluated, moreover for the task 1, we have created 5 task and the corresponding execution time is founded. Similarly for task 2 and task 4 average value of the execution time is taken.

The speed-up of the Hybrid Algorithm is shown in the Figure 7. The speed-up of the Hybrid algorithm increases when the number of task increased based on the time. Comparison is made with the ACO algorithm and the speed-up of ACO is evaluated based on the taks which has been given as the input. It shows clearly that the speed-up of the hybrid algorithm and ACO is increasing steadily for the number of tasks and when the more and tasks have been given as the input the speed-up is more in Hybrid Algorithm than in ACO. This is because the search operation is performed in the Hybrid algorithm by the cuckoo search.

Table 2. Task creation time

\begin{tabular}{|c|l|l|l|}
\hline \multicolumn{5}{|c|}{ TASK CREATION TIME } \\
\hline S1.No & Task 1 & Task 2 & Task 4 \\
\hline 1 & 0.0330 & 0.0334 & 0.0340 \\
\hline 2 & 0.0337 & 0.0357 & 0.0369 \\
\hline 3 & 0.0320 & 0.0321 & 0.0322 \\
\hline 4 & 0.0331 & 0.0346 & 0.0351 \\
\hline 5 & 0.0338 & 0.0301 & 0.0341 \\
\hline
\end{tabular}


International Journal of Information Technology Convergence and Services (IJITCS) Vol.2, No.4, August 2012

\begin{tabular}{|c|l|l|l|}
\hline Total & 0.1656 & 0.1659 & 0.1729 \\
\hline Average & 0.03312 & 0.03318 & 0.0348 \\
\hline
\end{tabular}

Table 3. Result Retrieval time

\begin{tabular}{|c|l|l|l|}
\hline \multicolumn{5}{|c|}{ RESULT RETRIEVAL TIME } \\
\hline Sl.No & Task 1 & Task 2 & Task 4 \\
\hline 1 & 0.0100 & 0.0104 & 0.0105 \\
\hline 2 & 0.0102 & 0.0103 & 0.0107 \\
\hline 3 & 0.0104 & 0.0110 & 0.0105 \\
\hline 4 & 0.0103 & 0.0104 & 0.0122 \\
\hline 5 & 0.0101 & 0.0105 & 0.0108 \\
\hline Total & 0.0510 & 0.0526 & 0.0547 \\
\hline Average & 0.0102 & 0.01052 & 0.01094 \\
\hline
\end{tabular}

Table 4. Total time

\begin{tabular}{|l|l|l|l|}
\hline \multicolumn{4}{|c|}{ TOTAL TIME } \\
\hline Sl.No & Task 1 & Task 2 & Task 4 \\
\hline 1 & 4.2042 & 4.2083 & 4.2113 \\
\hline 2 & 4.2021 & 4.2036 & 4.2052 \\
\hline 3 & 4.2030 & 4.2034 & 4.2036 \\
\hline 4 & 4.2142 & 4.2125 & 4.2380 \\
\hline 5 & 4.2112 & 4.2122 & 4.2133 \\
\hline Total & 21.0347 & 21.0400 & 21.0714 \\
\hline Average & 4.20694 & 4.2080 & 4.21428 \\
\hline
\end{tabular}


International Journal of Information Technology Convergence and Services (IJITCS) Vol.2, No.4, August 2012

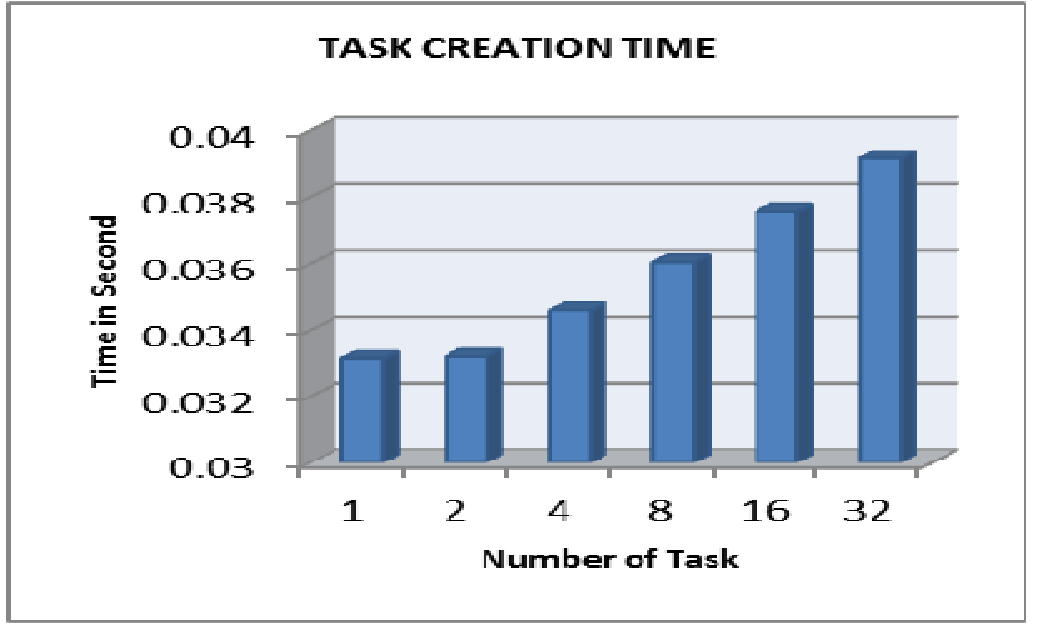

Figure 5. Task creation time

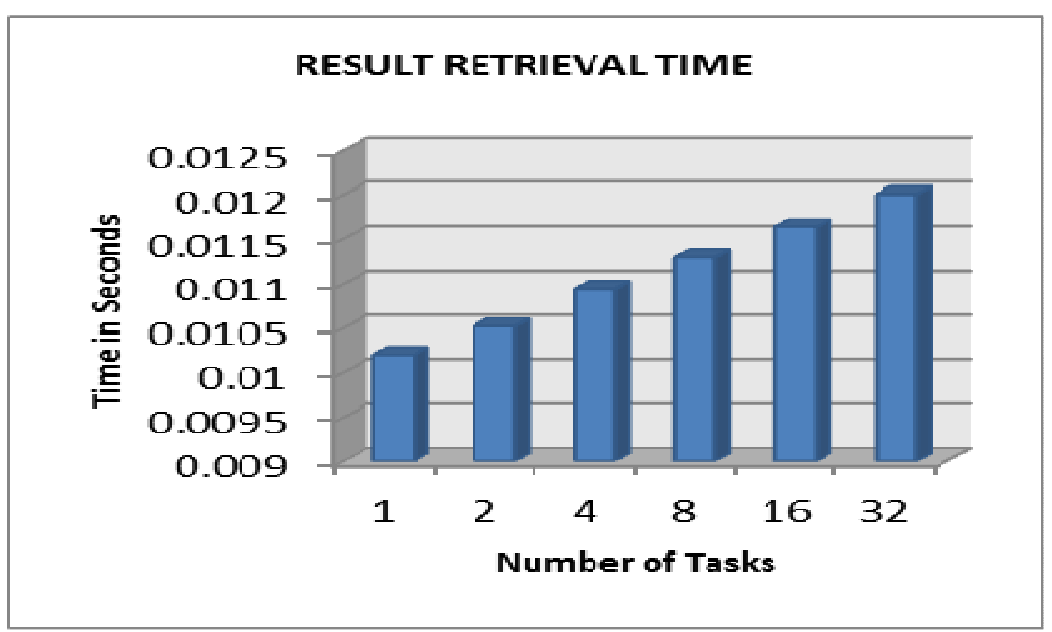

Figure 6. Result Retrieval time

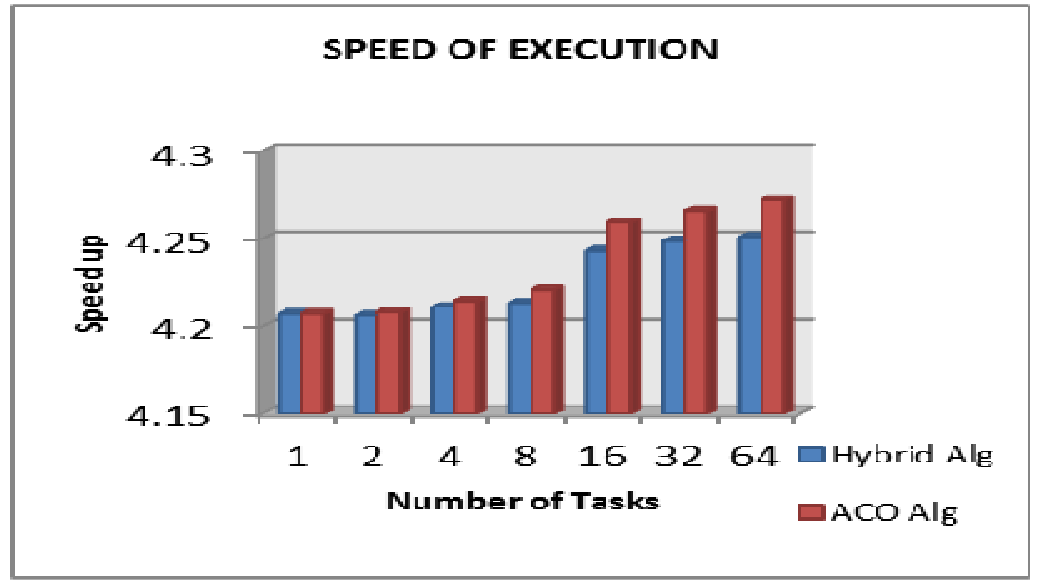

Figure 7. Speed of Execution 


\section{Conclusion}

Job scheduling is a type of combinatorial optimization problem, which can be used to validate the heuristic algorithms. In this paper, we have presented a Hybrid algorithm for job scheduling by combining the merits of ACO and Cuckoo search. The experimental analysis shows that as the number of tasks is increased, the time taken for the creation of tasks and result retrieval is also increased. In future we plan to apply the hybrid algorithm for job scheduling in scientific and high power computing to minimize the completion time and resource consumed.

\section{References}

1. Surekha, Sumathi,'PSO and ACO based approach for solving combinatorial Fuzzy Job Shop Scheduling”,Int. J. Comp. Tech. Appl., Vol 2 (1), 112-120 [2010].

2. Fabrizio Ferrandi, "Ant Colony Heuristic for Mapping and Scheduling Tasks and Communications on Heterogeneous Embedded Systems",Pier Luca Lanzi, IEEE Transactions on computer-Aided Design of Intergrated Circuits and Systems, vol 29 no.6, [2010].

3. Suchang Guo, Hong-Zhong Huang,"Grid Service Reliability Modeling and Optimal Task Scheduling Considering Fault Recovery",IEEE Transactions on Realiability vol.60,no.1 [2011].

4. Qi Tan, Hua-Ping Chen,"Two-agent scheduling on a single batch processing machine with nonidentical job sizes",Artificial Intelligence, Management Science and Electronic Commerce (AIMSEC),[2011].

5. Tavakkoli-Moghaddam,Azarkish,"A new hybrid mutli-objective Pareto archive PSO algorithm for a bi-objective job shop scheduling problem",Elsevier Expert Systems with Applications [2011].

6. Chang Wook Ahn, Jinung An,'Estimation of particle swarm distribution algorithms: Combining the benefits of PSO and EDAs,Elsevier Information Sciences [2010].

7. Xueming Yang, Jiangye Yuan,"An improved WM method based on PSO for electric load forecasting",Elsevier Expert Systems with Applications [2010].

8. Changseok Bae, Wei-Chang Yeh, Elsevier Expert Expert Systems with Applications [2010],'Feature selection with Intelligent Dynamic Swarm and Rough Set".

9. Sha, Hsing-Hung Lin,"A Multi-objective PSO for job-shop scheduling problems",Elsevier Expert Systems with Applications [2010].

10. Qian Tao, Hui-you Chang,"A rotary Chaotic PSO algorithm for trustworthy scheduling of a grid workflow",Elsevier Computers \& Operations Research [2011].

11. Xia-Min Hu, Jun Zhang, "SamACO: Variable Sampling Ant Colony Optimization Algorithm for Continuous Optimization"IEEE Transactions on systems, man, and cybernetics vol.40. no.6 [2010].

12. Zhiqiang Zhang, Jing Zhang, Shujuan Li, "A Modified Ant Colony Algorithm for the Job Shop Scheduling Problem to Minimize Makespan",IEEE Explore [2010].

13. Zhi-Hui Zhan, Jun Zhang, “An Efficient Ant Colony System Based on Receding Horizon Control for the Aircraft Arrival Sequencing and Scheduling Problem", IEEE Transactions on intelligent transaction systems. Vol. 11, no.2 [2010]. 